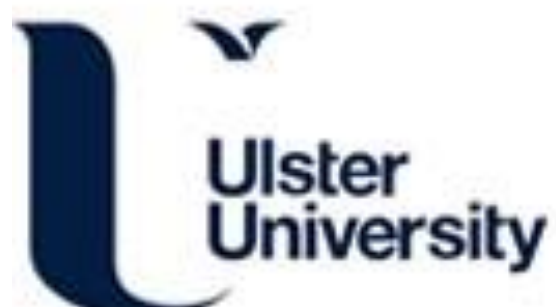

\section{A Bayesian filter approach to modelling human movement patterns for first responders within indoor locations}

Furey, E., Curran, K., \& McKevitt, P. (2011). A Bayesian filter approach to modelling human movement patterns for first responders within indoor locations. In F. Xhafa, L. Barolli, \& M. Koppen (Eds.), Unknown Host Publication (pp. 729-734). IEEE Computer Society. https://doi.org/10.1109/INCoS.2011.14

Link to publication record in Ulster University Research Portal

\section{Published in:}

Unknown Host Publication

Publication Status:

Published (in print/issue): 01/12/2011

DOI:

10.1109/INCoS.2011.14

\section{Document Version}

Publisher's PDF, also known as Version of record

\section{General rights}

Copyright for the publications made accessible via Ulster University's Research Portal is retained by the author(s) and / or other copyright owners and it is a condition of accessing these publications that users recognise and abide by the legal requirements associated with these rights.

\section{Take down policy}

The Research Portal is Ulster University's institutional repository that provides access to Ulster's research outputs. Every effort has been made to ensure that content in the Research Portal does not infringe any person's rights, or applicable UK laws. If you discover content in the Research Portal that you believe breaches copyright or violates any law, please contact pure-support@ulster.ac.uk. 


\title{
A Bayesian filter approach to modelling Human Movement Patterns for First Responders within Indoor Locations
}

\author{
Eoghan Furey, Kevin Curran, Paul Mc Kevitt \\ Faculty of Computing \& Engineering, \\ University of Ulster, Derry, Northern Ireland \\ Email: furey-e1@email.ulster.ac.uk
}

\begin{abstract}
The arrival of new devices and techniques has brought tracking out of the investigation stage and into the wider world. Using Wi-Fi signals is an attractive and reasonably affordable option to deal with the currently unsolved problem of widespread tracking in an indoor environment. Here we present a system called HABITS (History Aware Based Indoor Tracking System) which aims at overcoming weaknesses in existing Real Time Location Systems (RTLS) by using approach of making educated guesses about future locations of humans. The primary research question that is foremost is whether the tracking capabilities of existing RTLS can be improved automatically by knowledge of previous movement especially in the short term in the case of emergency first responders by the application of a combination of artificial intelligence approaches, a key contributor being Bayesian filters. We conclude that HABITS improves on the standard Ekahau RTLS in term of accuracy (overcoming black spots), latency (giving position fixes when Ekahau cannot), cost (less $A P s$ are required than are recommended by Ekahau) and prediction (short term predictions are available from HABITS). These are features that no other indoor tracking system currently provides and could provide crucial in future emergency first responder incidents.
\end{abstract}

Keywords: indoor location tracking, indoor positioning, first responder systems, wireless networks

\section{INTRODUCTION}

Our fire service personnel have an unenviable job. Frequently, their primary task at times is to enter a burning building equipped only with air, two-way radios and laden down with heavy outfits. The increasing fire and smoke intensity along with the possible destruction of escape paths can result in life threatening situations. There therefore exists a requirement to develop real-time location tracking systems to assist fire-fighters and other personnel in navigating a building safely. Recent advances in technology and integrated electronics have now made such a system feasible. What is needed to achieve such a system is some wearable device which will help pinpoint the current location of each rescue team member for an incident command post outside the building in addition to providing emergency exit guidance to each team member and a "homing" signal to guide searchers in finding any fire-fighters in trouble.
It turns out that to achieve tracking some sort of frame of reference is normally required and a number of waypoints need to be established. In satellite positioning the satellites themselves provide these waypoints, their position relative to each other and relative to the ground is known, therefore location of an unknown device can be calculated relative to these. The same principle applies in the majority of positioning systems. Problems arise when these reference points are either too few in number, or those that are there do not have a clear line of sight to the object being tracked. Occlusion, attenuation, reflection and refraction are the cause of many errors in RTLS. While no solution works perfectly in all environments, within reason, almost anything can be tracked to any desired resolution if enough resources are available. These resources can be quantified in terms of financial cost and vastly reduce the scalability of the RTLS. Innovative methods are required to improve accuracy levels and to enable positioning to be achieved for a reasonable cost in terms of time and infrastructure. The recent proliferation of mobile communications devices is enabling tracking and analysis of large groups of people to be conducted. Where available, this data is being mined searching for patterns and trends which facilitate prediction $[1,2,3]$.

This paper outlines a system which aims at overcoming weaknesses in existing RTLS by using the approach of making educated guesses about people's location. The hypothesis here is that knowledge of a person's historical movement habits enables future location predictions to be made in the short, medium and long term. The research questions that are foremost are whether the tracking capabilities of existing RTLS can be improved automatically by knowledge of historical movements and by the application of a combination of artificial intelligence approaches. A practical application of our system would be for use by first-responders inside buildings.

\section{PREDICTING LOCATION}

To accurately position an object, a degree of intelligence is required. Outdoors, using GPS traces to try and learn next location has been attempted by Han [4] for someone 
on foot and in [5] for vehicles on a road. More recently data gathered from mobile phone records has been mined to try and find patterns of movement which could be used to try and make next location predictions [6]. Indoors, this is a largely under researched area, however a number of 'smart environments' have been set up such as the work [1]. Here specific sensors on doors were utilised to provide movement patterns. A Hidden Markov Model (HMM) and a Neutral Network (NN) were applied to the data and successful predictions were made. Since around 2006, due to its commercial value, most of the leading research in next location prediction is not being conducted by academic institutions and therefore everything that has been learnt may not be in the public domain. Ashbrook and Starner [7], used a markov chain model and k-means clustering algorithm to attempt to predict future movement. They clustered GPS data to find significant locations and then built a first and second order markov models using location as state to try and predict future movement. It is possible to create an $n^{\text {th }}$ order Markov model where probability of the next state is dependent not only on the current state but on the previous n-1 states. For some examples, considering the $2^{\text {nd }}$ order can yield more accurate results as in the case of probability of transition from $\mathrm{A} \rightarrow \mathrm{B}$ is $70 \%$ but the probability of transitioning from $\mathrm{B} \rightarrow \mathrm{A} \rightarrow \mathrm{B}$ is $81 \%$. This could be explained by a situation where A was a Shop and B was Home. If the shop was on the main road from Home then the probability of going from A to B (Shop to Home) is $70 \%$. However, if the journey started at home and went to the shop, return to home could be more probable (perhaps getting something for dinner?). This demonstrates a situation when higher order models are useful and give extra information. It raises the question of which order of model is suitable for prediction. Ashbrook and Starner [7] conclude that this depends on the quantity of data available. Other factors affecting their probabilities were due to the large distances travelled and the fact that their tests took place outdoors. They also found that changes in routine would take a long time to show up in their model and they suggested a possible method of weighting certain updates, but warned that this could lead to model that was somewhat skewed. Han [4] attempts to build upon the work of [7] by using a Self Organising Map (SOM) as a means of learning without pre-knowledge. To use a supervised learning method to learn patterns of movements, pre-knowledge of the person is required, however a SOM can overcome this.

One of the first research projects that considered future movement was Microsoft Research's RADAR project [8]. This was the first significant attempts to track indoors using 802.1 Wi-Fi signals. Due to the severe problem of signal attenuation it was difficult to get an accurate fix on position using Received Signal Strength (RSS) measurements alone. Position was occasionally reported in locations that were not possible or at least highly unlikely. An effort to overcome these problems is described in Bahl and Padmanabhan [9]. They concluded that the next location position should be close to the last reported one. Their Viterbi-like tracking algorithm deals with a situation of when two physically separate locations are close together in signal space (due to aliasing). The shortest path is depicted in bold. The likely trajectory is calculated based on the previous unambiguous location and a guess of somewhere in between the two is given. They tested the Viterbi-like approach against an NNSS (Nearest Neighbour in Signal Space) and an NNSS-AVG where the three nearest neighbours in signal space were averaged to estimate location and it was found to significantly outperform the others. Median distance error for NNSS $(3.59 \mathrm{~m})$ and NNSS-AVG $(3.32 \mathrm{~m})$ are $51 \%$ and $40 \%$ worse, respectively compared with Viterbi [9]. Hidden Markov Models (HMM) are a popular technique which have been successfully applied in numerous different fields. The application of HMM to speech recognition has been examined by Rabiner [10]. In speech recognition predicting the next possible words can greatly increase accuracy. Rabiner examined HMM from their simplest form (discrete Markov chain) to more sophisticated approaches such as continuous density models and those of variable duration. These techniques have been in widespread use for many years in speech recognition software. Computational biology is another field that has seen widespread application of predictive machine learning. The use of HMM for gene prediction in sequences of DNA has been reviewed by Birney [11]. A new method for predicting the secondary structure of RNA using HMM was proposed by Yoon and Vaidynathan [12]. They demonstrated very accurate, secondary structure prediction using their proposed model with a low computational cost. [13] converted algorithms normally used as "branch prediction techniques for current high performance microprocessors" to handle next context prediction of a person. These were applied to previously gathered behaviour patterns. The predictors were stimulated with patterns of behaviour of people walking indoors as the workload.

\section{MODELLING A TRACKING SYSTEM}

The three main components of HABITS (History Aware Based Indoor Tracking System) are (1) a connected graph, (2) a discrete Bayesian filter and (3) a set of logic rules. A key focus of this work involves combining these three methods in a novel way, enabling predictions of human movement habits. These predictions overcome the latency of updates from currently available systems and enable them to make predictions of likely future movement. The underlying principle of our approach involves representing the movement areas as a graph which in turn is represented by a number of matrices; 
incidence, distance and transition. These constraints show where it is possible for a user to go and where not, the distance between points of interest (for our purposes) and eventually represent the probability of going from one area to another. The graph enables us to represent this information in mathematical terms and as numbers which may then be processed by a machine/computer. Methods of modelling the travel environment exist and of these, a graph structure closely represents the travelable paths. The nodes in the graph can be positioned to represent areas of interest, decision points or places where the user stops. In between these locations are the paths that may be travelled between. The paths may be considered as edges and those locations of interest could be the nodes/vertices of a connected graph. The graph structure clearly represents the connections between nodes and therefore areas in the real building. It shows which locations are connected either directly or indirectly. When studying a building plan or road map this information is normally clear to see. However, in a new location, different methods need to be used to identify these areas of interest. Areas where a user stops for some reason may be thought of as base nodes. Stopping for reasons such as sleep, eating, call of nature or work are some of the main reasons why humans would habitually stop at the same location. While for many people these may be in the same room or adjacent rooms, in the developed world, relatively large houses exist and these functions often occur in a number of rooms with travel paths between. Examples of rooms could be bedroom, bathroom and living room. Movement between rooms is often only possible by one or two different routes.

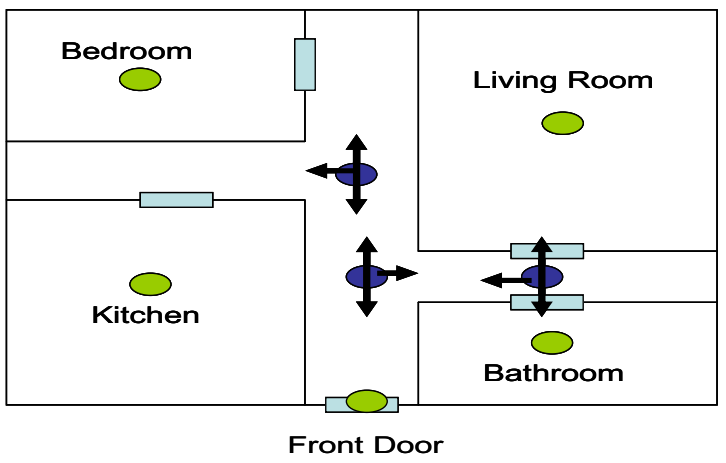

Figure 1: Node positions in house

The layout of a typical house (in the developed world) may be represented as a connected graph (see Figure 2), the green nodes represent stopping locations and the blue nodes represent decision points. Learning the locations of these points can be done automatically in a number of ways, all of which require an underlying tracking system to be installed. Learning these locations can be performed automatically by computers. One method of achieving this is to plot the locations where there was a significant delay between movements. These would indicate the areas where a person was stationary. Even within the same room these points are not all likely to be in the exact same location. To extract wait nodes from a large number of estimates, clustering techniques are used to group the updates together, revealing the main stopping locations. When the nodes have been discovered and coded with numbers for names (Figure 2) they may be represented as an $\mathrm{n} \times \mathrm{n}$ adjacency matrix where $\mathrm{n}$ is the number of nodes and the matrix details specific information about the graph.

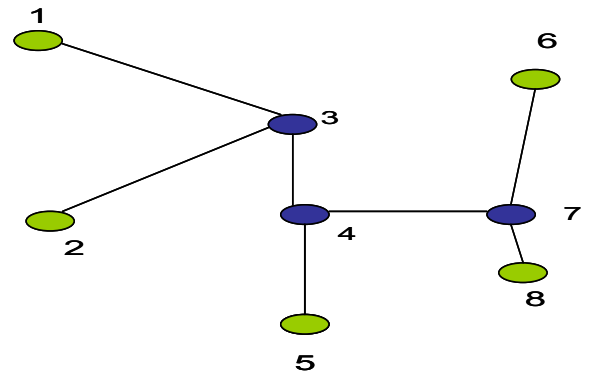

Figure 2: Node names replaced with numbers

Figure 3 shows the adjacency matrix corresponding to the connected graph which in turn corresponds to the node positions in the sample house (Figure 2). If a connection exists between the nodes then in the matrix location ij which represents the connection from $i$ to $j$ place a 1 , if no connection exists then place a zero. This enables the paths between nodes to be represented mathematically and the matrix can easily be processed by a computer program.

\begin{tabular}{|lll|l|l|l|l|l|l|l|}
\hline & $\mathbf{1}$ & $\mathbf{2}$ & $\mathbf{3}$ & $\mathbf{4}$ & $\mathbf{5}$ & $\mathbf{6}$ & $\mathbf{7}$ & $\mathbf{8}$ \\
$\mathbf{1}$ & 0 & 0 & 1 & 0 & 0 & 0 & 0 & 0 \\
$\mathbf{2}$ & 0 & 0 & 1 & 0 & 0 & 0 & 0 & 0 \\
$\mathbf{3}$ & 1 & 1 & 0 & 1 & 0 & 0 & 0 & 0 \\
$\mathbf{4}$ & 0 & 0 & 1 & 0 & 1 & 0 & 1 & 0 \\
$\mathbf{5}$ & 0 & 0 & 0 & 1 & 0 & 0 & 0 & 0 \\
$\mathbf{6}$ & 0 & 0 & 0 & 0 & 0 & 0 & 1 & 0 \\
$\mathbf{7}$ & 0 & 0 & 0 & 1 & 0 & 1 & 0 & 1 \\
$\mathbf{8}$ & 0 & 0 & 0 & 0 & 0 & 0 & 1 & 0 \\
\hline
\end{tabular}

Figure 3: Adjacency matrix for nodes in sample house

When the node locations have been discovered and the distance between two nodes is known, travel time between nodes may also be calculated automatically by the underlying tracking system. Average walking or travelling speed for each user is estimated by using the formula speed $=$ distance/time. Knowledge of the relative 
travel times between nodes facilitates generation of a distance matrix with distances between each node being calculated based on average user speed. The distance matrix values are in the same positions in the matrix as the 1's are in the adjacency matrix. A transition matrix showing the probabilities of travelling from one node to the other is built up by monitoring the person's travel through the nodes. At any time along the chain, only the current location gives the probability of going to the next location. A simple Markov chain like this gives some idea of the next node but alone it would not be enough to model real human movement habits. Raising the order of the model to consider the previous two nodes would help in some locations but [5] proved this needs to be done with a large dataset which takes a considerable time to generate. Maintaining a separate transition matrix for each day and/or each time period would improve the accuracy slightly but the system would not be expandable to a large area due to becoming overly complex. To predict the most likely next location with a useful degree of accuracy requires more than just a simple one state Markov chain. The underlying tracking system gives the initial location, bel $\left(\mathrm{x}_{\mathrm{t}-1}\right)$. The transition matrix provides the belief, bel $\left(\mathrm{x}_{\mathrm{t}}\right)$ when combined with the information in the Perceptual Model and the System Dynamics. This outputs the probability of moving to the next node when given just the previous one and no other information. HABITS uses more information than just that provided by the first order markov chain. As a Bayesian filter only works for instances that hold to the markov assumption (meaning only a single order model), a great deal of information is being left out about commonly travelled paths or sequences of nodes. Froehlich and Krumm [5] found that the more nodes they had information about, (previously travelled) the higher the chances of predicting their final location. If an order ( 3 for example) markov model was used, then for some paths, the predicted location probability would be much higher, however it would also take into account shorter journeys and could have sequences like 2-4-2 which would include changing direction completely. Taking into account higher order models makes the calculations overcomplicated. The notion of preferred paths (PP), however allows for the same information to be gathered without keeping track of every path. As part of the definition of a habit, it states that they are routines of behaviour that are repeated regularly. Preferred paths are stored as a vectors and may be temporally linked to a specific time period if required (some would be more frequently travelled at particular times than others). When on a preferred path, the information is used to increase the accuracy of the future location estimate. If we assume it is known that Node 1 was the node visited at time, $t_{-3}$. This would now give a sequence of nodes 1-3-4 leading up to the decision point. The preferred path vector for that particular sequence would be the probability of going to node 5 or 6 from that point. We assume that preferred paths only consider movement to new nodes and do not consider backward movement. We now have a vector showing:

$P P_{-}$Node4 $=\left[\begin{array}{l}0.66 \\ 0.33\end{array}\right]$

This tells us that when the sequence of nodes visited was 1-3-4, the likelihood of being on the preferred path 1-3-45 is 0.66 and the likelihood of being on the preferred path $1-3-4-6$ is 0.33 . The method combining these two probabilities multiplies them together and adds the results to the initial belief from the Bayesian filter. The new belief gives a much higher probability of going to node 5 next than of going to node 6 . A last influencing factor to be considered in some instances is a rule that takes into account when people change their habits depending on who they were with. In largely populated environments certain people's movements have an influence on other peoples. If, for example, the habit is going for lunch it may be that a particular person is a common factor in most locations. This is discovered by checking to see if people travel routes matched up temporally and if so, was one dominant over the other? When this is the case, a rule is applied in the same manner as the preferred paths, influencing the prediction. HABITS combines a number of different elements to produce future location predictions. The inputs to the Bayesian filter include the Motion Model showing where it is possible to go in the next step, the Sensor model giving the accuracy of the updates from the underlying tracking system, the learnt Historical belief and the location updates from the base system. When the filter has all the necessary information to give a prediction, it is run through a set of rules to improve the accuracy of its estimates. When a mobile device is tracked by the Ekahau RTLS and HABITS is applied, it can still be tracked when it is no longer within line of sight (LOS) of three or more Access Points (AP). This is normally the minimum required for accurate localisation. The highest frequency rate of position updates from the Ekahau RTLS has been found to be $5 \mathrm{~s}$. HABITS can dramatically improve standard location tracking systems and provide accurate updates of where the user is located.

\section{MOVEMENT INDOORS WITH HABITS}

Ekahau RTLS is a commercial WiFi localisation system. For Ekahau to work, an existing 802.11 wireless network must be in place in the test area. Ekahau contains a number of components. The Ekahau Positioning Engine (EPE acts as a server controlling all location updates. It needs to be located on a server which has access to the existing WLAN. Once the server is in place, the Ekahau Site Survey (ESS) model is created. A number of steps 
are required for this. First, a map (Jpeg file) of each floor is uploaded to the ESS application. Figure 4 shows a signal strength map for the University MS Building. The dark green colour indicates areas of good signal strength where tracking capability should be accurate. These heat maps also show areas of accurate signal strength are weak. These are target areas for HABITS.

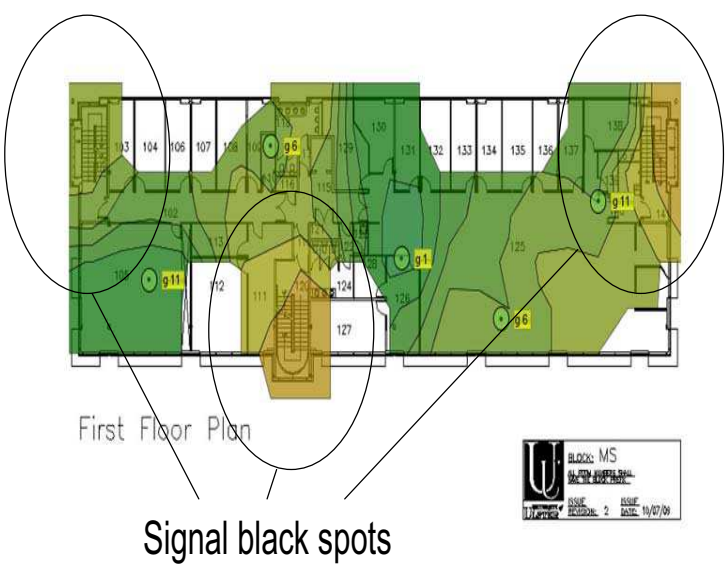

Figure 4: First Floor Plan - Signal Strength map

In order to collect historical movement data a topological map of the test area is created. A topological map is one which consists of a number of nodes representing places of interest which are connected by edges representing paths where a user may travel. Each of these zones can be considered to be a node in a connected graph. The positioning of these zones is a manual process based on expert knowledge of where a user is likely to stop and areas where they would pass through often. Also used are locations where a user has a number of options of where next to travel. The locations of these zones relative to one another can now be represented as an adjacency matrix and hence a connected graph. To do this each node in the graph representing a zone is given a unique ID between 1 and $n$, were $n$ is the number of zones. By querying the EPE the list of zones is retrieved and each zone is allocated a unique ID. For the two floors in the MS building there are 19 zones in total. Using the zone map, an adjacency matrix of size $\mathrm{n} \times \mathrm{n}$ is manually created. The corresponding zone-node list allows all zone data from the EPE to be manipulated as if each zone was the node in the connected graph. Figure 5 shows a connected graph representation of the two floors in the ISRC. The edges between nodes show paths that may be travelled and represent the movements of Wi-Fi tracked people in the building. The numbers on the nodes are those used by the zone to node conversion table. Knowledge of where the user is, whether they are in motion or not and the exact time are essential for HABITS to function. This is the only live information that HABITS processes. Once the live user information is received, HABITS checks the matrices to see what constraints on movement exist. Combining these constraints with the data from Ekahau allows an initial probability prediction to be made from the transition matrix. For instance, if an update is now received from the Ekahau RTLS. This update is not in a zone so using the nearest neighbour search the closest node is found to be node 16 . Given that the last zone entered update was from the wait node 18, HABITS now possesses three bits of new information.

$\mathrm{x}_{\mathrm{t}}=$ node $16, \mathrm{x}_{\mathrm{t}-1}=$ node $18 \&$ user Eoghan is 'in-motion'.

From this new information HABITS can offer only a general prediction of the next possible node that will be visited. Eight different options are possible at this stage. Node 16 is not a 'wait node' therefore HABITS does not consider this to be the end of the current journey. The options at node 16 are listed in Table 1.

\begin{tabular}{|c|c|c|c|c|c|c|c|c|}
\hline $\begin{array}{c}\text { Node } \\
\text { no }\end{array}$ & 15 & 13 & 12 & 11 & 14 & 17 & 19 & 5 \\
\hline $\operatorname{Bel}(x t)$ & 0.27 & 0.05 & 0.05 & 0.01 & 0.05 & 0.05 & 0.01 & 0.01 \\
\hline
\end{tabular}

While node 15 is given as having a much higher probability that the other possible next nodes, 0.27 is too low to make any kind of reasonable prediction so HABITS must wait for more information from Ekahau. If another location update is received between nodes 15 and 4. HABITS knows from the previous bel(xt) that node 15 was one of the last possible nodes, therefore, even though node 15 is slightly closer to the update than node 4 (from the k-nearest neighbours search, 15 closest, followed by 4 ), it chooses node 4 as the next node from which to make a calculation. At this point due to a lack of other options and the fact that HABITS has information about the previous four nodes 18-16-15-4, a fairly confident prediction can be made that the next node to be visited is will be node 5 . When a third update is received which is very close to node 5 .

Table 2: Probabilities from the transition matrix at node 5

\begin{tabular}{|l|l|l|l|l|l|}
\hline Node Number & $\mathbf{3}$ & $\mathbf{2}$ & $\mathbf{6}$ & $\mathbf{7}$ & $\mathbf{8}$ \\
\hline Bel(xt) & $\mathbf{0 . 1 7}$ & $\mathbf{0 . 0 2}$ & $\mathbf{0 . 3 1}$ & $\mathbf{0 . 1 5}$ & $\mathbf{0 . 0 2}$ \\
\hline
\end{tabular}

Given the previous nodes sequence, the distance from the third update to node 5 and the previous prediction, HABITS carries out the next prediction based on node 5 as its current node. As node 5 is also a transition node, possible predictions from the transition matrix give five possible next nodes as Table 2 shows. At this stage no prediction can be made as node next node has a clear higher probability. Probabilities change when the preferred paths for the current time period are checked. Here, as the time period is equal to 'lunch' and the previous node sequence is quite extensive at this stage, 
two nodes, 3 and 6 now possess a significantly hìghe probability that the other possible nodes.

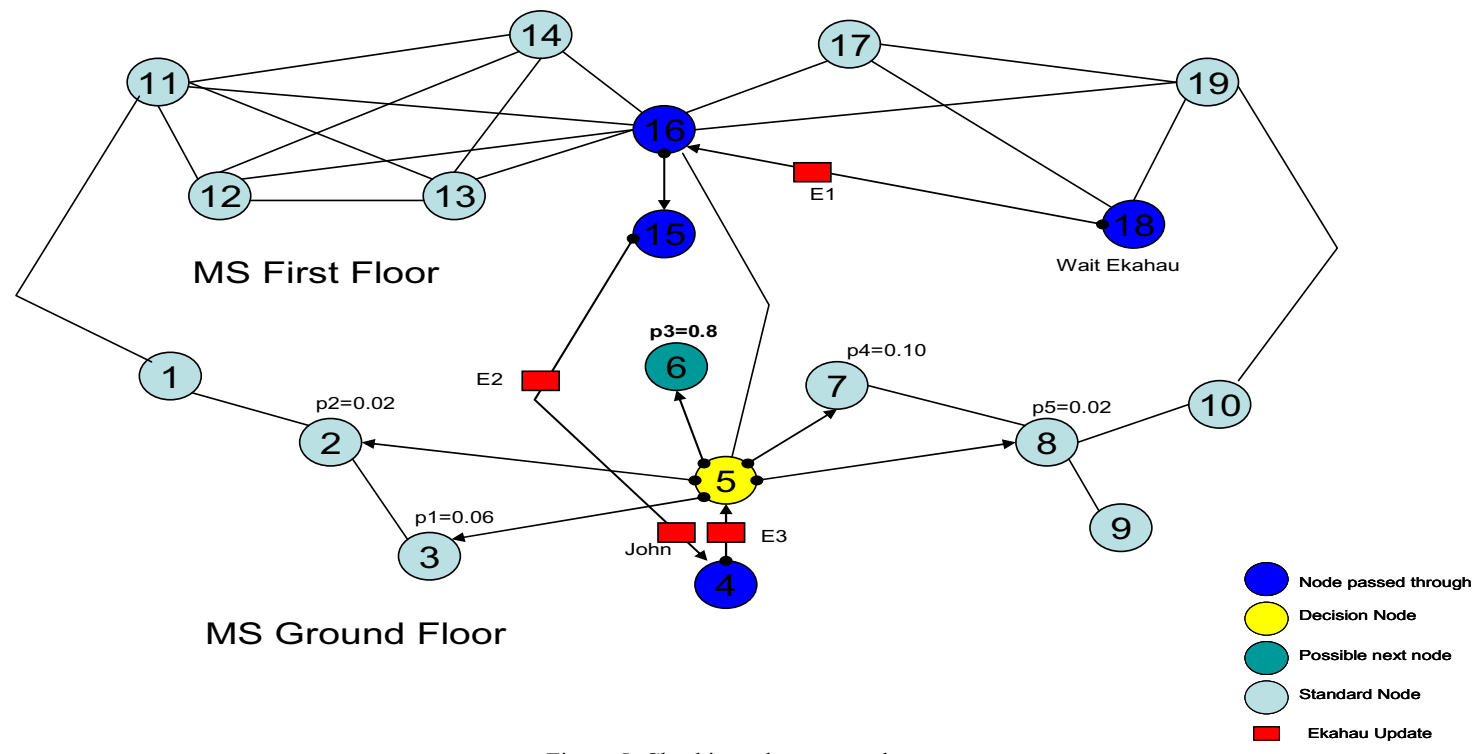

Figure 5: Checking other users rule

HABITS can now say with over $80 \%$ confidence that the next node to be visited will be either node 3 or node 6 . To further increase the accuracy of the predictions, HABITS checks to see if there are any other users in the area. Figure 5 depicts a position update being received from John. Examining the preferred path of John reveals that John does not go to node 3. As the paths of Eoghan and John are regularly together, combining John's 'preferred path' probability with Eoghan's gives a new prediction that Eoghan will go to node 6 with $80 \%$ confidence.

\section{CONCLUSION}

This paper outlines a system (History Aware Based Indoor Tracking System) which aims at overcoming weaknesses in existing RTLS by using the approach of making educated guesses about people's future locations. We conclude that HABITS improves on the standard Ekahau RTLS in term of accuracy (overcoming black spots), latency (giving position fixes when Ekahau cannot), cost (less APs are required than are recommended by Ekahau) and prediction (short term predictions are available from HABITS). These are features that no other indoor tracking system currently provides and could provide crucial in emergency first responder incidents.

\section{REFERENCES}

1. Petzold, J., Bagci, F., Trumler, W. et al.: Next Location Prediction within a Smart Office Building. 1st International Workshop on Exploiting Context Histories in Smart Environments at the 3rd Int Conference on Pervasive Computing, Munich, Germany, May, (2005)

2. Vintan, L., Gellert, A., Petzold, J. et al.: Person Movement Prediction using Neural Networks. (2004)

3. Gellert, A., \& Vintan, L.: Person Movement Prediction using Hidden

Markov Models. Studies in Informatics and control, 15 (2006) 17

4. Han, S. J.: Learning and Predicting User's Movements for Intelligent Location-Based Services. (2004)

5. Froehlich, J., \& Krumm, J.: Route Prediction from Trip Observations. SAE SP, 2193 (2008) 53

6. González, M. C., Hidalgo, C. A., Barabási, A. L.: Understanding Individual Human Mobility Patterns. Nature, 453 (2008) 779-782

7. Ashbrook, D., \& Starner, T.: Learning Significant Locations and Predicting User Movement with GPS. Wearable Computers,

2002.(ISWC 2002). 6th International Symposium on, (2002) 101-108

8. Bahl, P., \& Padmanabhan, V. N.: RADAR: An in-Building RF-Based User Location and Tracking System. INFOCOM 2000. Nineteenth Annual Joint Conference of the IEEE Computer and Communications Societies. Proceedings. IEEE, 2 (2000) 775-784 vol.2

9. Bahl, P., Padmanabhan, V. N., Balachandran, A.: Enhancements to the RADAR User Location and Tracking System. Microsoft, (2000)

10.Rabiner, L. R.: A Tutorial on HMM and Selected Applications in Speech Recognition. Proceedings of the IEEE, 77 (1989) 257-286

11. Birney, E.: Hidden Markov Models in Biological Sequence Analysis. IBM Journal of Research and Development, 45 (2001) 449-454

12. Yoon, B. J., \& Vaidyanathan, P.: RNA Secondary Structure Prediction using Context-Sensitive Hidden Markov Models. (2004) S2/7/INV-S2/7/1-4

13. Petzold, J., Bagci, F., Trumler, W. et al.: Hybrid Predictors for Next Location Prediction. LECTURE NOTES IN COMPUTER SCIENCE, 4159 (2006) 125 